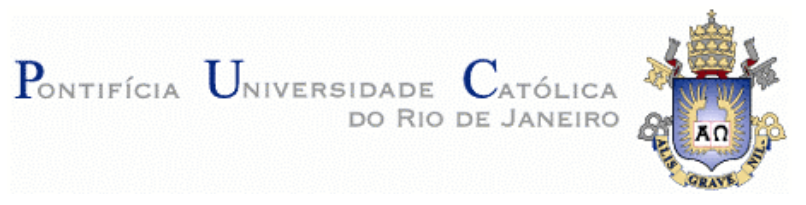

Eduardo Costa da Silva

Desenvolvimento de Transdutor Baseado na Fase da Magnetoimpedância Gigante para Medição de Campos Biomagnéticos

Dissertação de Mestrado

Dissertação apresentada ao Programa de Pós-Graduação em Metrologia da PUC-Rio como requisito parcial para obtenção do título de Mestre em Metrologia. Área de Concentração: Metrologia para Qualidade e Inovação.

Orientadora: Profa. Elisabeth Costa Monteiro, Dra. Co-Orientador: Prof. Carlos Roberto Hall Barbosa, Dr.

Rio de Janeiro abril de 2010 
Eduardo Costa da Silva

\section{Desenvolvimento de Transdutor Baseado na Fase da Magnetoimpedância Gigante para Medição de Campos Biomagnéticos}

Dissertação apresentada como requisito parcial para obtenção do grau de Mestre pelo Programa de Pós-Graduação em Metrologia do Centro Técnico Científico da PUC-Rio. Aprovada pela Comissão Examinadora abaixo assinada.

Profa. Elisabeth Costa Monteiro, Dra. Orientadora

Programa de Pós Graduação em Metrologia (Pós-MQI) Pontifícia Universidade Católica do Rio de Janeiro (PUC-Rio)

Prof. Carlos Roberto Hall Barbosa, Dr. Co-Orientador

Programa de Pós Graduação em Metrologia (Pós-MQI) Pontifícia Universidade Católica do Rio de Janeiro (PUC-Rio)

Prof. Jean Pierre von der Weid, PhD Departamento de Engenharia Elétrica (ELE) Pontifícia Universidade Católica do Rio de Janeiro (PUC-Rio)

Prof. Maurício Nogueira Frota, PhD

Programa de Pós Graduação em Metrologia (Pós-MQI) Pontifícia Universidade Católica do Rio de Janeiro (PUC-Rio)

Prof. Marcio Nogueira de Souza, Dr. Programa de Engenharia Biomédica (PEB) Universidade Federal do Rio de Janeiro (UFRJ)

Prof. José Eugênio Leal, PhD

Coordenador Setorial do Centro Técnico Científico - PUC-Rio 
Todos os direitos reservados. É proibida a reprodução total ou parcial do trabalho sem autorização da universidade, do autor e do orientador.

\section{Eduardo Costa da Silva}

Graduado em Engenharia Elétrica (ênfases em Eletrônica e Controle de Processos) e em Engenharia de Controle e Automação, pela Pontifícia Universidade Católica do Rio de Janeiro (PUC-Rio), em 2008.

Ficha Catalográfica

Silva, Eduardo Costa da

Desenvolvimento de Transdutor Baseado na Fase da Magnetoimpedância Gigante para Medição de Campos Biomagnéticos / Eduardo Costa da Silva ; orientadora: Elisabeth Costa Monteiro ; co-orientador: Carlos Roberto Hall Barbosa. - 2010.

231 f. : il. (color.) ; $30 \mathrm{~cm}$

Dissertação (Mestrado em Metrologia) - Pontifícia Universidade Católica do Rio de Janeiro, Centro Técnico Científico, Rio de Janeiro, 2010.

Inclui bibliografia

1. Metrologia - Teses. 2. Metrologia. 3. Biometrologia. 4. Magnetômetro. 5. Biomagnetismo. 6. Magnetocardiografia. 7. Magnetoimpedância Gigante. 8. Fase da impedância. I. Monteiro, Elisabeth Costa. II. Barbosa, Carlos Roberto Hall. III. Pontifícia Universidade Católica do Rio de Janeiro. Centro Técnico Científico. Programa de Pós-Graduação em Metrologia. Área de Concentração: Metrologia para Qualidade e Inovação. IV. Título. 
Dedico este trabalho a meus pais, Eduardo e Beatriz, por apoiarem e incentivarem minha formação acadêmica, e pela paciência e compreensão nos momentos difíceis. 


\section{Agradecimentos}

À Deus, por iluminar meus pensamentos e guardar meu caminho.

À Nossa Senhora de Fátima, por permitir meu nascimento e guiar meus passos.

À Ciência, por ser a lanterna que permite que o homem enxergue os caminhos escondidos na escuridão.

À Curiosidade, por ser a pilha que permite o funcionamento da lanterna chamada Ciência. Os questionamentos geram a pesquisa, não as respostas.

À PUC-Rio, por acreditar na pesquisa científica como instrumento de desenvolvimento social e evolução humana.

Ao CNPq e a FINEP, pelos auxílios financeiros concedidos.

Aos meus pais, Eduardo e Beatriz, pelo amor, pela confiança, por acreditarem em minhas escolhas, pelo apoio incondicional, pela compreensão nos momentos de ausência, por acreditar na importância do estudo como ferramenta de formação pessoal e transformação social, e pelo apoio à minha formação acadêmica.

À minha avó, Maria, pelo amor, carinho e atenção.

À minha família e amigos, por respeitarem o caminho que escolhi e compreenderem os momentos de ausência. 
Ao Professor Luiz Antônio Pereira de Gusmão, por ter me apresentado a linha de pesquisa que propiciou o desenvolvimento desta dissertação e introduzido-me aos melhores orientadores que eu poderia encontrar. Por ter colaborado de forma incondicional para o desenvolvimento deste trabalho, por ter acreditado em minhas idéias e auxiliado no caminho que as tornou reais. Por enxergar soluções práticas para meus devaneios teóricos. Mas, principalmente, por ter se tornado um grande amigo!

A meus orientadores, Professora Elisabeth Costa Monteiro e Professor Carlos Roberto Hall Barbosa, por não limitarem meu campo de pesquisa e apoiarem todas as minhas sugestões. Por estimularem meu desenvolvimento e não cercearem o desenvolvimento de minhas idéias. Por acreditarem na ciência brasileira. A Professora Elisabeth pelo otimismo e ao Professor Hall pelo realismo.

Ao Professor Fernando Luiz de Araújo Machado, pela acolhida em minha visita ao Departamento de Física da UFPE, pelos esclarecimentos e por fornecer as amostras GMI utilizadas em minha pesquisa.

Aos Professores do PósMQI da PUC-Rio, pelos frutificantes ensinamentos.

Ao coordenador do PósMQI da PUC-Rio, Professor Maurício Frota, pelo apoio a linha de pesquisa em Biometrologia e por propiciar um ambiente de desenvolvimento científico transdisciplinar, conforme a Metrologia preconiza e necessita, através da junção e colaboração entre mestrandos provenientes de diversas áreas de formação acadêmica.

Aos colegas do Laboratório de Biometrologia, Flávia Pompéia e Daniel Louzada, pelo apoio e colaboração.

Às secretárias e ex-secretárias do PósMQI da PUC-Rio, Márcia Ribeiro, Paula e Isabel, pelo apoio e esclarecimentos. 
Aos Professores do Departamento de Engenharia Elétrica e do Departamento de Engenharia de Controle e Automação da PUC-Rio, pela sólida formação acadêmica.

À equipe de suporte técnico do Departamento de Engenharia Elétrica da PUC-Rio, em especial aos técnicos Manuel Ramos Martins, Isnarde Antonio Ernesto e Evandro Costa dos Reis, por permitirem meu acesso a oficina e por terem um estoque de componentes eletrônicos superior ao da Farnell.

Ao Professor Gilson Gomes Vieira, atual Coordenador da Fundação Planetário do Rio de Janeiro, por ter despertado meu interesse pela pesquisa científica, ainda durante meu $2^{\circ}$ grau, e por quase ter me levado a cursar Astronomia.

À equipe da Agência PUC-Rio de Inovação, em especial Shirley Coutinho, Taís e Márcio, por acreditarem e estimularem a integração universidade-empresa, por promoverem cursos e palestras sobre propriedade intelectual no ambiente da PUC-Rio, por permitirem que pesquisadores da universidade informem-se sobre aspectos relativos à propriedade intelectual, e por serem um instrumento de transformação de invenções em inovações. 


\section{Resumo}

Costa da Silva, Eduardo; Monteiro, Elisabeth Costa; Barbosa, Carlos Roberto Hall. Desenvolvimento de Transdutor Baseado na Fase da Magnetoimpedância Gigante para Medição de Campos Biomagnéticos. Rio de Janeiro, 2010. 231p. Dissertação de Mestrado - Programa de Pós-Graduação em Metrologia. Pontifícia Universidade Católica do Rio de Janeiro.

Esta dissertação tem por objetivo o desenvolvimento de um transdutor magnético baseado no efeito da Magnetoimpedância Gigante (GMI - Giant Magnetoimpedance), destinado à medição de campos biomagnéticos. A motivação deste trabalho advém da inexistência de um sistema de baixo custo capaz de medir satisfatoriamente campos biomagnéticos, operando à temperatura ambiente e em plena conformidade com os princípios biometrológicos. Por sua vez, a metodologia experimental empregada iniciou-se pela medição e minuciosa análise das características de módulo e fase de sensores GMI em função do campo magnético, verificando-se suas dependências em relação ao comprimento das amostras e ao nível CC e frequência da corrente de excitação. Também, investigou-se a histerese e a homogeneidade das amostras GMI. Em seguida, foram idealizados circuitos eletrônicos para condicionamento e leitura das amostras GMI, destacando-se a estrutura desenvolvida que possibilita a leitura gradiométrica e algumas configurações inovadoras que permitem tanto a amplificação da sensibilidade de fase dos sensores GMI quanto a homogeneização das características de fase. Os resultados obtidos para o melhor transdutor magnético GMI desenvolvido, o qual é baseado na fase da impedância, apresentou uma elevada sensibilidade $(250 \mathrm{mV} / \mathrm{nT})$, indicando ganhos superiores a 1500 vezes quando comparado a protótipos baseados no módulo e, consequentemente, possibilitando alcançar novos patamares de resolução. Como conclusão ressaltase o grande potencial do magnetômetro GMI desenvolvido, caracterizado por seu baixo custo e elevada sensibilidade, quanto a sua aplicabilidade na medição de campos magnéticos ultra-fracos e, em especial, de campos biomagnéticos. Assim, o presente desenvolvimento contribui para a difusão das técnicas diagnósticas baseadas na medição de campos biomagnéticos, no ambiente clínico-hospitalar.

\section{Palavras-chave}

Metrologia; Biometrologia; Magnetômetro; Biomagnetismo; Magnetocardiografia; Magnetoimpedância Gigante; Fase da Impedância. 


\section{Abstract}

Costa da Silva, Eduardo; Monteiro, Elisabeth Costa (Advisor); Barbosa, Carlos Roberto Hall (Co-Advisor). Development of a Transducer Based on the Phase of the Giant Magnetoimpedance for the Measurement of Biomagnetic Fields. Rio de Janeiro, 2010. 231p. M.Sc. Dissertation Programa de Pós-Graduação em Metrologia. Pontifícia Universidade Católica do Rio de Janeiro.

This M.Sc. dissertation aims at developing a magnetic field transducer based on the Giant Magnetoimpedance (GMI) effect to be applied on the measurement of biomagnetic fields. The motivation of this work stems from the lack of a low cost system capable of adequately measuring biomagnetic fields, operating at room temperature and in full conformity with the biometrological principles. On the other hand, the experimental methodology began by measuring and carefully analyzing the impedance magnitude and phase characteristics of GMI sensors as a function of the magnetic field, verifying their dependencies on the length of the samples and on the DC level and frequency of the excitation current. It was also investigated the hysteresis and the homogeneity of GMI samples. In turn, the electronic circuits for conditioning and reading of GMI samples were developed, highlighting the developed structure that enables a gradiometric reading and some innovative configurations that allow both the enhancement of the impedance phase sensitivity of GMI sensors and the homogenization of their impedance phase characteristics. The results obtained for the best GMI magnetic transducer developed, which is based on the impedance phase, showed a high sensitivity $(250 \mathrm{mV} / \mathrm{nT})$, indicating gains of more than 1500 times when compared to magnitude-based prototypes and, consequently, reaching much better resolution levels. In conclusion it is emphasized the great potential of the developed GMI magnetometer, characterized by its low cost and high sensitivity, as to its applicability in measuring ultra-weak magnetic fields and in particular biomagnetic fields. Thus, the present development contributes to the spread of diagnostic techniques based on the measurement of biomagnetic fields, in clinical and hospital environments.

\section{Keywords}

Metrology; Biometrology; Magnetometer; Biomagnetism; Magnetocardiography; Giant Magnetoimpedance; Impedance Phase. 


\section{Sumário}

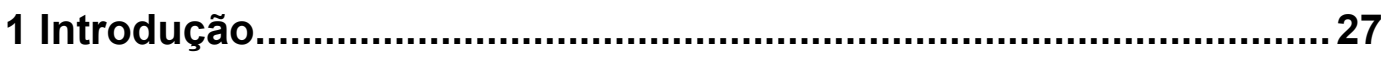

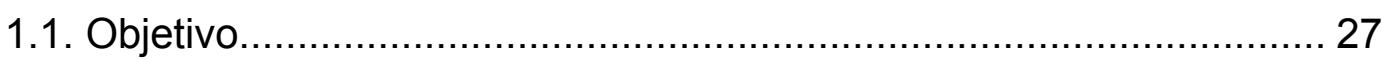

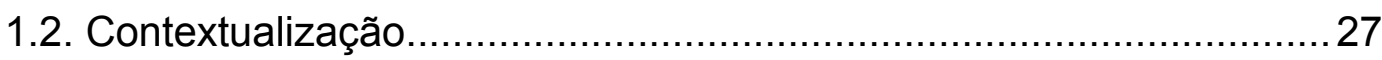

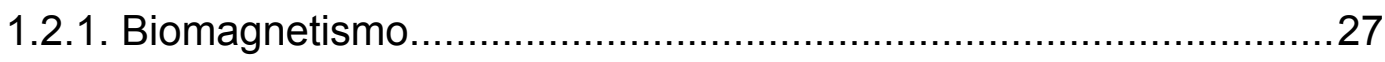

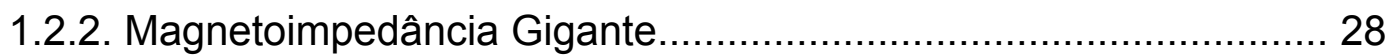

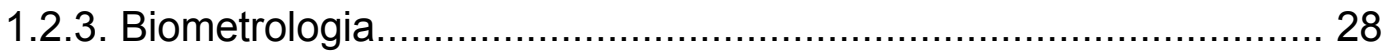

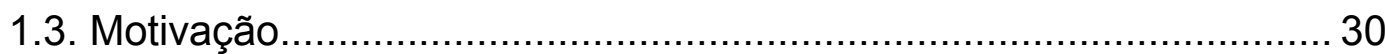

1.4. Confiabilidade Metrológica de Equipamentos Eletromédicos............ 32

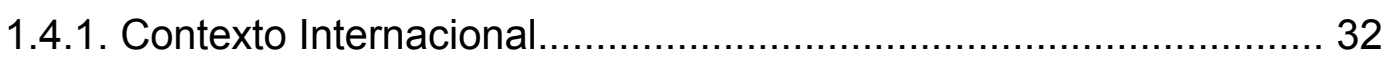

1.4.1.1. BIPM: Estrutura e Aspectos Relevantes.................................. 32

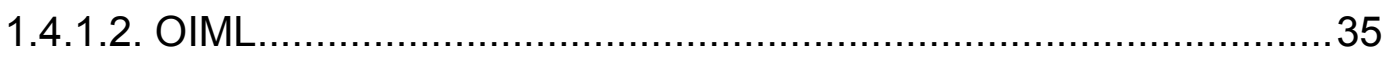

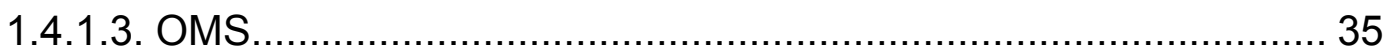

1.4.1.4. IAEA

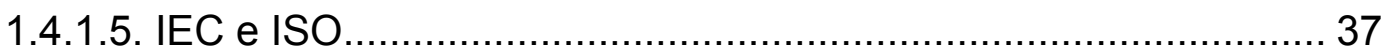

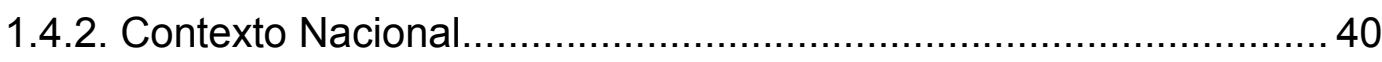

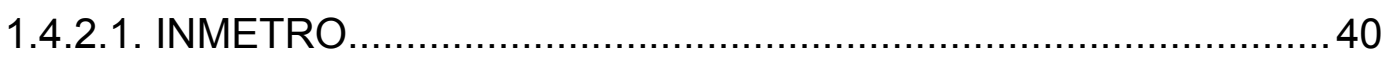

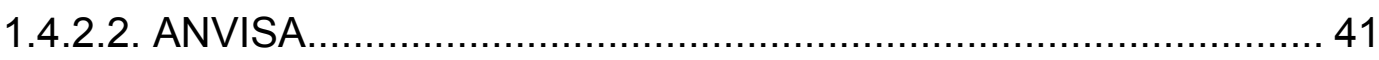

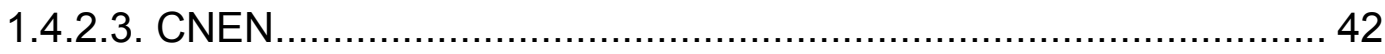

1.4.2.4. ABNT

1.5. Conservação da Grandeza Densidade de Fluxo Magnético............... 44

1.5.1. Grandezas Magnéticas no Brasil - INMETRO_..............................45

1.6. Sistema de Unidades e Conversões................................................ 46

1.7. Considerações: Terminologia e Outros Aspectos.............................. 47

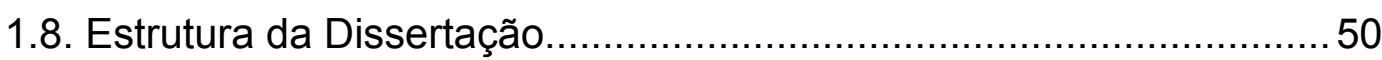

2 Fundamentos do Biomagnetismo.................................................. 53

2.1. Magnetobiologia versus Biomagnetismo............................................53

2.2. Fontes de Campo Biomagnético.................................................... 54

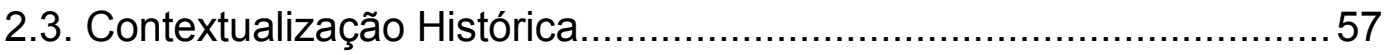




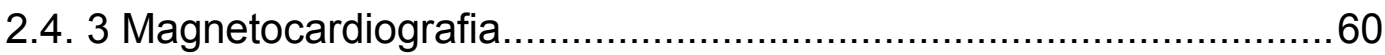

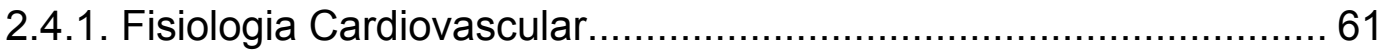

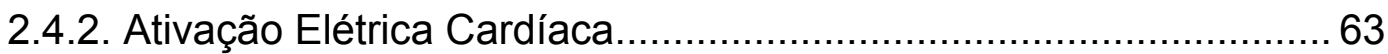

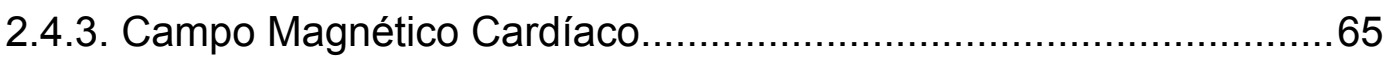

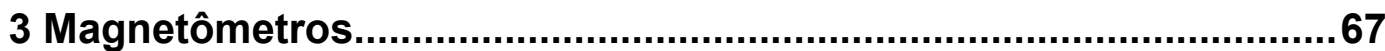

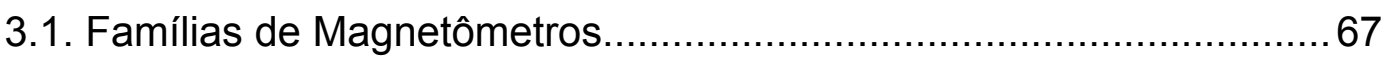

3.1.1. Superconducting Quantum Interference Device (SQUID)..............70

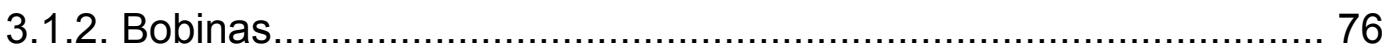

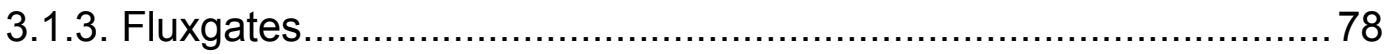

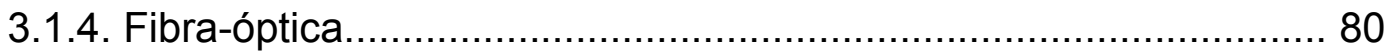

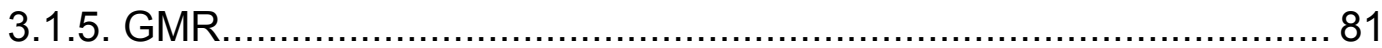

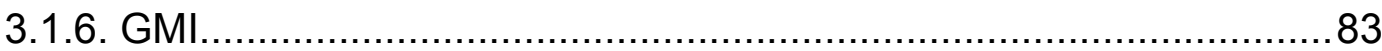

3.2. Redução do Ruído Magnético Ambiental e da Interferência

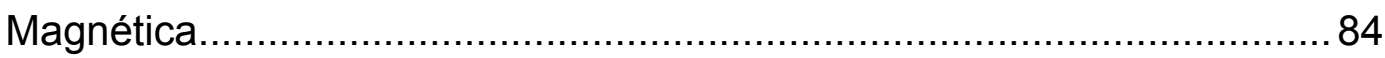

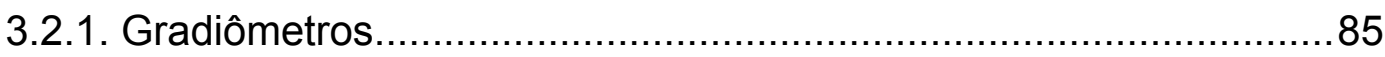

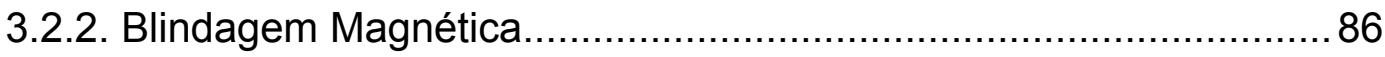

4 Magnetoimpedância Gigante (GMI)................................................. 91

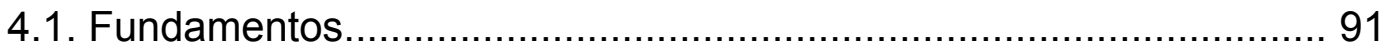

4.2. Magnetoimpedância Longitudinal (LMI)...................................... 92

4.3. Estrutura dos Domínios Magnéticos.............................................. 94

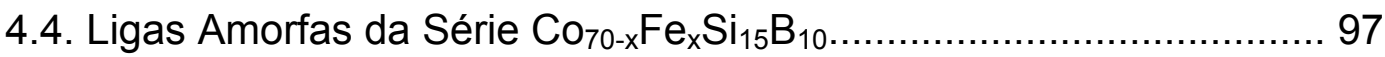

4.5. Efeito GMI por Faixa de Frequências............................................ 98

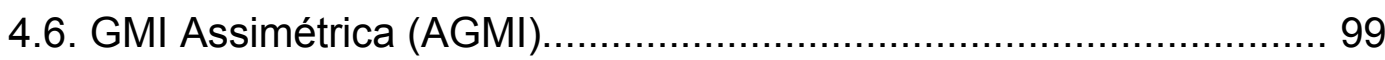

4.6.1. AGMI Induzida por Corrente CC............................................ 100

4.6.2. AGMI Induzida por Campo Magnético CA................................. 102

4.6.3. AGMI Induzida por "Exchange Bias".......................................... 103

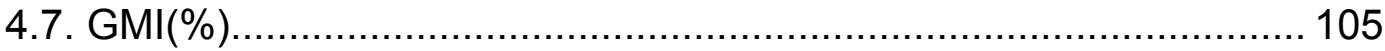

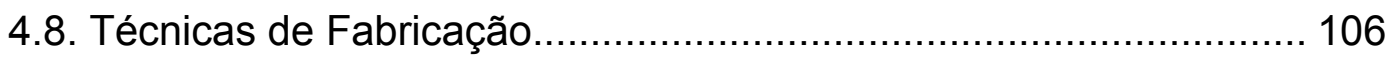

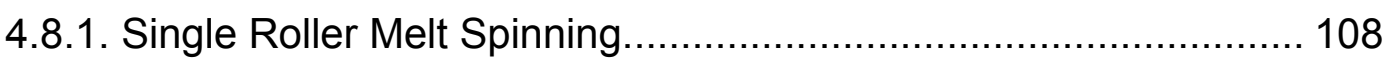

5 Caracterização Experimental das Fitas GMI..................................111

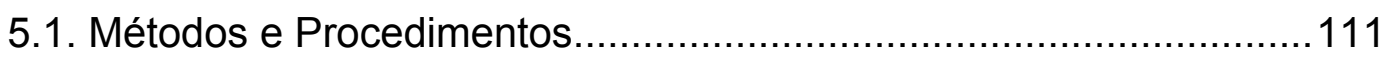




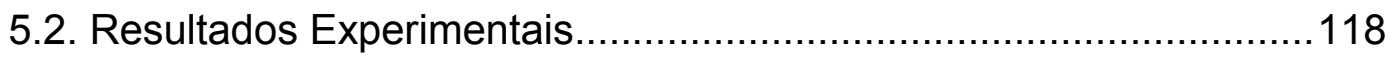

5.2.1. Influência da Frequência da Corrente de Excitação...................... 119

5.2.1.1. Módulo da Impedância......................................................... 119

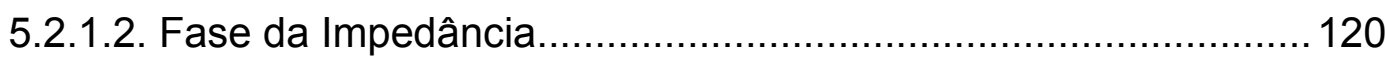

5.2.1.3. Análise da Influência da Frequência da Corrente de Excitação. 122

5.2.2. Influência do Nível CC da Corrente de Excitação......................... 124

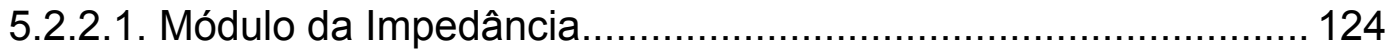

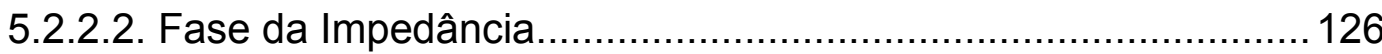

5.2.2.3. Análise da Influência do Nível CC da Corrente de Excitação.... 127

5.2.3. Influência do Comprimento das Fitas GMI................................. 128

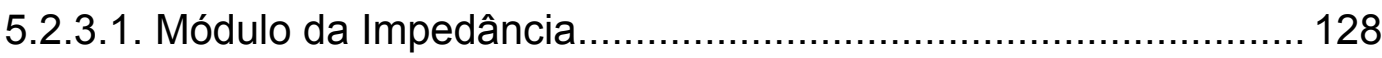

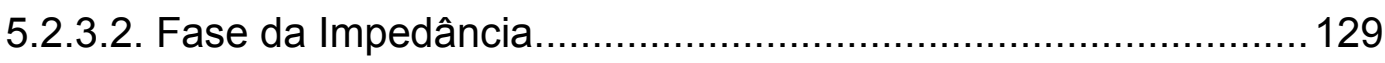

5.2.3.3. Análise da Influência do Comprimento das Fitas GMI.............. 131

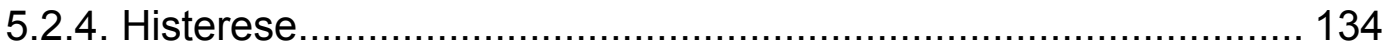

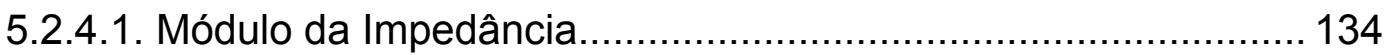

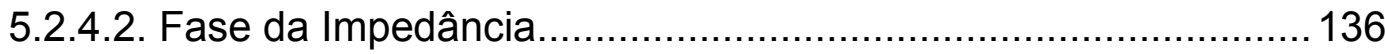

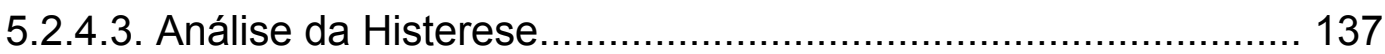

5.2.5. Homogeneidade das Amostras GMI........................................ 138

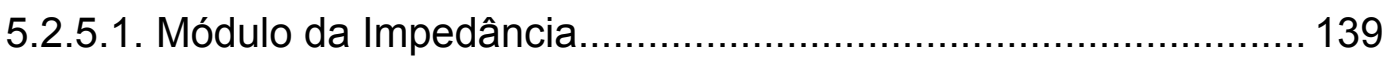

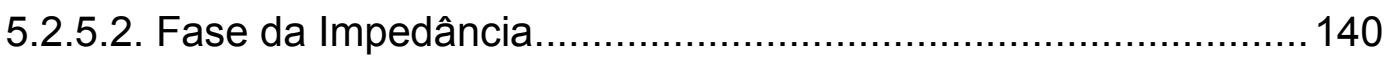

5.2.5.3. Análise da Homogeneidade das Amostras GMI.......................142

6 Desenvolvimento do Transdutor Magnético GMI.............................145

6.1. Utilização das Fitas GMI como Elementos Sensores -

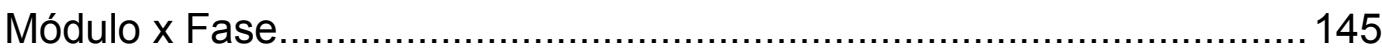

6.2. Circuitos Eletrônicos de Condicionamento e Leitura das Fitas GMI.146

6.2.1. Circuito de Detecção da Variação de Módulo da Impedância...... 146

6.2.2. Circuito de Detecção da Variação de Fase da Impedância........... 148

6.2.3. Simulações - Comparação: Módulo x Fase..................................149

6.3. Transdutor de Pressão Baseado nas Propriedades de Fase da Impedância do Efeito GMI..............................................................152

6.4. Amplificação da Sensibilidade de Fase.........................................154

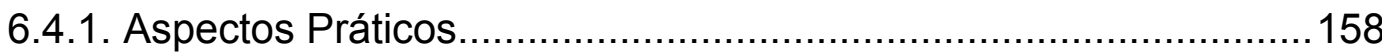


6.4.2. Versão \#1 do Circuito de Amplificação da Sensibilidade de Fase 160

6.4.3. Versão \#2 do Circuito de Amplificação da Sensibilidade de Fase 162

6.5. Montagem da Versão Simplificada do Protótipo............................ 174

7 Configuração Gradiômétrica e Leitura Triaxial................................ 181

7.1. Configurações Gradiômétricas Propostas....................................181

7.1.1. Gradiômetro GMI com Fitas Homogêneas................................181

7.1.2. Gradiômetro GMI com Fitas Heterogêneas - Circuito

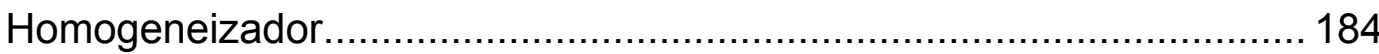

7.2. Configuração do Transdutor Magnético GMI para Leitura Triaxial.. 192

7.3. Protótipo Idealizado do Transdutor Magnético 193

8 Discussões, Conclusões e Trabalhos Futuros............................... 199

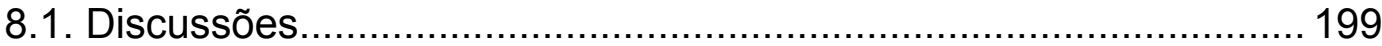

8.1.1. Caracterização Experimental das Amostras GMI....................... 199

8.1.2. Desenvolvimento do Transdutor GMI........................................201

8.1.3. Propriedade Intelectual - Prospecção Tecnológica...................... 204

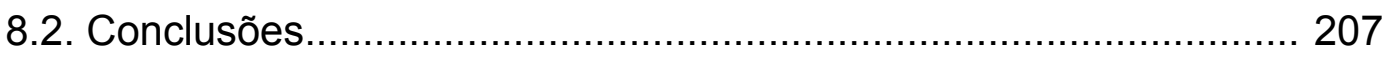

8.3. Trabalhos Futuros................................................................. 208

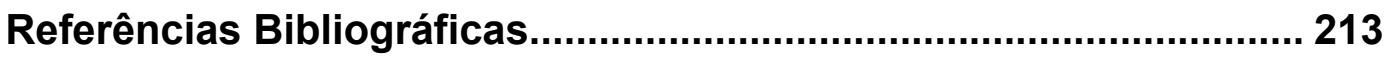




\section{Lista de Figuras}

Figura 1 - Taxa de mortalidade no mundo, por causa mortis e gênero, de acordo com a Organização Mundial da Saúde (OMS). 30

Figura 2 - Percentual de óbitos (número de óbitos por habitante) provenientes de doenças do aparelho circulatório no Brasil, entre 1996 e 2007

Figura 3 - Cronologia, com enfoque nas grandezas eletromagnéticas, do Sistema Internacional de Unidades (SI): da criação do BIPM à introdução do mol

Figura 4 - Diagrama de blocos das inter-relações do TC 62 e seus subcomitês com os demais TC's e SC's do IEC e da ISO. 38

Figura 5 - Fontes de campo magnético localizadas no ser humano....... 54

Figura 6 - Intensidade e Frequência de campos biomagnéticos. 55

Figura 7 - Comparativo entre as densidades de fluxo magnético dos campos biomagnéticos e as produzidas por fontes de ruído ambiental. .56

Figura 8 - Problema Direto versus Problema Inverso............................. 56

Figura 9 - Localização do coração humano (gettyimages.com).............. 61

Figura 10 - O coração e suas cavidades................................................61

Figura 11 - Estruturas responsáveis pela ativação elétrica cardíaca.......64

Figura 12 - Registro das ondas P-QRS-T ao longo de um ciclo da ativação elétrica cardíaca

Figura 13 - Sinal de MCG (ciclo PQRST) obtido por um magnetômetro SQUID 66

Figura 14 - Representação esquemática de uma junção Josephson...... 72

Figura 15 - Componentes básicos dos elementos sensores dos SQUID's RF e DC 
Figura 16 - Representação esquemática do SQUID. 75

Figura 17 - Medições magnetocardiográficas utilizando sistema SQUID multicanal.. 76

Figura 18 - Configuração do primeiro magnetômetro capaz de medir um MCG (1962).

Figura 19 - Configuração esquemática de um transdutor magnético do tipo Fluxgate.

Figura 20 - Curva de magnetização com Histerese. 79

Figura 21 - Princípio de funcionamento de um Fluxgate. 79

Figura 22 - Configuração esquemática de um transdutor magnético a Fibra-óptica 80

Figura 23 - Diposição das camadas do sensor GMR "spin-valve" e variação da magnetização em função do campo externo, em (a) $\mathrm{H}=0$, as magnetizações de FF e FL estão orientadas perpendicularmente, $R=R(0)$; (b) $H \neq 0$, as magnetizações de FF e FL estão paralelamente orientadas e com sentidos iguais, $R<R(0)$; (c) $H \neq 0$, as magnetizações de FF e FL estão paralelamente orientadas e com sentidos opostos, $R>R(0)$; (d) $\mathrm{H}$ muito intenso, rompe a fixação de $F F, R<R(0)$. 82

Figura 24 - Configurações gradiômétricas (SQUID): (A) ordem zero detecta todas as linhas de campo que atravessam a bobina, (B) $1^{\mathrm{a}}$ ordem e (C) $2^{\mathrm{a}}$ ordem. 85

Figura 25 - (a1) e (a2) Blindagens por correntes parasitas (eddy currents) e sem utilização de $\mu$-metal, (b) Blindagem padrão ( $\mu$-metal-alumínio- $\mu$-metal), (c) Blindagem de $\mu$-metal e alumínio de alta atenuação (mais camadas de $\mu$-metal), (d) Blindagem Supercondutora.

Figura 26 - Câmara blindada BMSR-2 em construção (PTB, Berlim, Alemanha), com a primeira camada de $\mu$-metal instalada 89

Figura 27 - Câmara blindada BMSR-2 pronta para operação 90 
Figura 28 - Fator de blindagem (S) versus frequência (f) para as câmaras magneticamente blindadas que possuem os melhores fatores de atenuação, construídas em centros de pesquisa em biomagnetismo localizados na Alemanha (BMSR) e no Japão (COSMOS).

Figura 29 - Medição típica do efeito GMI. 92

Figura 30 - Configuração dos domínios magnéticos de (a) fitas amorfas com magnetostricção positiva, (b) fitas amorfas com magnetostricção negativa, e (c) fitas amorfas com magnetização positiva ou quase nula após tratamento térmico (annealing).

Figura 31 - Configuração da estrutura dos domínios magnéticos para uma fita GMI $\left(\mathrm{Co}_{66,5} \mathrm{Fe}_{3,5} \mathrm{Si}_{12} \mathrm{~B}_{18}\right)$...

Figura 32 - Configuração dos domínios magnéticos em fitas e fios GMI

Figura 33 - Configuração experimental para indução de AGMI, por corrente CC, numa amostra em forma de fio

Figura 34 - GMI(\%) em função do campo magnético externo, variando-se a intensidade e o sentido de $\mathrm{I}_{\mathrm{cc}}$ 101

Figura 35 - Configuração experimental para indução de AGMI por campo CA em amostras em forma de fio. 103

Figura 36 - Representação esquemática de uma fita amorfa, após recozimento em ar com aplicação de campo magnético 104

Figura 37 - Representação esquemática do processo "Single Roller Melt Spinning". 108

Figura 38 - Equipamento utilizado, na UFPE, para produção de amostras GMI pela técnica "Single Roller Melt Spinning". 109

Figura 39 - Detalhes do interior do equipamento apresentado na Fig. 38 109

Figura 40 - Diagrama de Blocos do sistema utilizado na caracterização das fitas GMI 
Figura 41 - Bobina de Helmholtz com a fita GMI posicionada em seu centro

Figura 42 - Curva de Histerese.

Figura 43 - Configuração do aparato para realização da solda de ponto - Vista 1

Figura 44 - Configuração do aparato para realização da solda de ponto - Vista 2

Figura 45 - Configuração do aparato montado para conexão por "contato mecânico" da fita GMI aos terminais elétricos - Vista 1... 117

Figura 46 - Configuração do aparato montado para conexão por "contato mecânico" da fita GMI aos terminais elétricos - Vista 2.... 117 Figura 47 - Módulo da impedância de uma fita GMI de $1 \mathrm{~cm}$ submetida a $i_{C}=[80+15 . \operatorname{sen}(2 \pi$.f.t) $] \mathrm{mA}$, onde $\mathrm{f}$ assume os valores $100 \mathrm{kHz}, 250 \mathrm{kHz}, 500 \mathrm{kHz}, 1 \mathrm{MHz}$ e $10 \mathrm{MHz}$ 119

Figura 48 - Módulo da impedância de uma fita $\mathrm{GMI}$ de $3 \mathrm{~cm}$ submetida a $\mathrm{i}_{\mathrm{C}}=[80+15 . \operatorname{sen}(2 \pi$.f.t) $] \mathrm{mA}$, onde f assume os valores $100 \mathrm{kHz}, 1 \mathrm{MHz}, 2 \mathrm{MHz}, 5 \mathrm{MHz}$ e $10 \mathrm{MHz}$

Figura 49 - Módulo da impedância de uma fita GMI de $5 \mathrm{~cm}$ submetida a $\mathrm{i}_{\mathrm{C}}=[80+15 . \operatorname{sen}(2 \pi$.f.t) $] \mathrm{mA}$, onde f assume os valores $100 \mathrm{kHz}, 500 \mathrm{kHz}, 1 \mathrm{MHz}, 2 \mathrm{MHz}, 5 \mathrm{MHz}$ e $10 \mathrm{MHz} \ldots . .120$

Figura 50 - Módulo da impedância de uma fita GMI de $15 \mathrm{~cm}$ submetida a $i_{C}=[80+15 . \operatorname{sen}(2 \pi . f . t)] m A$, onde $f$ assume os valores $2 \mathrm{MHz}, 5 \mathrm{MHz}$ e $10 \mathrm{MHz}$ 120

Figura 51 - Fase da impedância de uma fita GMI de $1 \mathrm{~cm}$ submetida a $i_{C}=[80+15 . \operatorname{sen}(2 \pi . f . t)] m A$, onde $f$ assume os valores $100 \mathrm{kHz}, 250 \mathrm{kHz}, 500 \mathrm{kHz}, 1 \mathrm{MHz}$ e $10 \mathrm{MHz}$ 121

Figura 52 - Fase da impedância de uma fita $\mathrm{GMI}$ de $3 \mathrm{~cm}$ submetida $a i_{C}=[80+15 . \operatorname{sen}(2 \pi$.f.t) $] \mathrm{mA}$, onde f assume os valores $100 \mathrm{kHz}, 250 \mathrm{kHz}, 500 \mathrm{kHz}, 750 \mathrm{kHz}, 1 \mathrm{MHz}$, $2 \mathrm{MHz}, 5 \mathrm{MHz}$ e $10 \mathrm{MHz}$. 
Figura 53 - Fase da impedância de uma fita $\mathrm{GMl}$ de $5 \mathrm{~cm}$ submetida a $\mathrm{i}_{\mathrm{C}}=[80+15 . \operatorname{sen}(2 \pi . \mathrm{f} . \mathrm{t})] \mathrm{mA}$, onde f assume os valores $100 \mathrm{kHz}, 250 \mathrm{kHz}, 500 \mathrm{kHz}, 750 \mathrm{kHz}, 1 \mathrm{MHz}$, $2 \mathrm{MHz}, 5 \mathrm{MHz}$ e $10 \mathrm{MHz}$. 121

Figura 54 - Fase da impedância de uma fita GMI de $15 \mathrm{~cm}$ submetida a $\mathrm{i}_{\mathrm{C}}=[80+15 . \operatorname{sen}(2 \pi$.f.t $)] \mathrm{mA}$, onde $\mathrm{f}$ assume os valores $2 \mathrm{MHz}, 5 \mathrm{MHz}, 10 \mathrm{MHz}, 15 \mathrm{MHz}$ e $20 \mathrm{MHz}$.

Figura 55 - Módulo da impedância de uma fita GMI de $1 \mathrm{~cm}$ submetida $\mathrm{a} \mathrm{i}_{\mathrm{C}}=\left[\mathrm{I}_{\mathrm{cC}}+15 . \operatorname{sen}(2 \pi .100 \mathrm{kHz} . \mathrm{t})\right] \mathrm{mA}$, onde $\mathrm{I}_{\mathrm{CC}}$ assume os valores $0 \mathrm{~mA}, 40 \mathrm{~mA}$ e $80 \mathrm{~mA}$. 124

Figura 56 - Módulo da impedância de uma fita GMI de $3 \mathrm{~cm}$ submetida $a \mathrm{i}_{\mathrm{C}}=\left[\mathrm{I}_{\mathrm{cc}}+15 . \operatorname{sen}(2 \pi .5 \mathrm{MHz} . \mathrm{t})\right] \mathrm{mA}$, onde $\mathrm{I}_{\mathrm{cc}}$ assume os valores $0 \mathrm{~mA}, 40 \mathrm{~mA}$ e $80 \mathrm{~mA}$

Figura 57 - Módulo da impedância de uma fita GMI de $5 \mathrm{~cm}$ submetida $a i_{C}=\left[I_{C C}+15 . \operatorname{sen}(2 \pi .100 \mathrm{kHz} . t)\right] \mathrm{mA}$, onde $\mathrm{I}_{\mathrm{CC}}$ assume os valores $0 \mathrm{~mA}, 40 \mathrm{~mA}$ e $80 \mathrm{~mA}$. 125

Figura 58 - Módulo da impedância de uma fita GMI de $15 \mathrm{~cm}$ submetida $\mathrm{a} \mathrm{i}_{\mathrm{C}}=\left[\mathrm{I}_{\mathrm{cC}}+15 \cdot \operatorname{sen}(2 \pi \cdot 10 \mathrm{MHz} \cdot \mathrm{t})\right] \mathrm{mA}$, onde $\mathrm{I}_{\mathrm{CC}}$ assume os valores $0 \mathrm{~mA}, 40 \mathrm{~mA}$ e $80 \mathrm{~mA}$.. 125

Figura 59 - Fase da impedância de uma fita GMI de $1 \mathrm{~cm}$ submetida $a i_{C}=\left[I_{C C}+15 . \operatorname{sen}(2 \pi .100 k H z . t)\right] m A$, onde $I_{C C}$ assume os valores $0 \mathrm{~mA}, 40 \mathrm{~mA}$ e $80 \mathrm{~mA}$ 126

Figura 60 - Fase da impedância de uma fita GMI de $3 \mathrm{~cm}$ submetida $a \mathrm{i}_{\mathrm{C}}=\left[\mathrm{I}_{\mathrm{cc}}+15 \cdot \operatorname{sen}(2 \pi \cdot 2 \mathrm{MHz} . \mathrm{t})\right] \mathrm{mA}$, onde $\mathrm{I}_{\mathrm{Cc}}$ assume os valores $0 \mathrm{~mA}, 40 \mathrm{~mA}$ e $80 \mathrm{~mA}$ 126

Figura 61 - Fase da impedância de uma fita GMI de $5 \mathrm{~cm}$ submetida $\mathrm{a} \mathrm{i}_{\mathrm{C}}=\left[\mathrm{I}_{\mathrm{CC}}+15 . \operatorname{sen}(2 \pi \cdot 100 \mathrm{kHz} . \mathrm{t})\right] \mathrm{mA}$, onde $\mathrm{I}_{\mathrm{CC}}$ assume os valores $0 \mathrm{~mA}, 40 \mathrm{~mA}$ e $80 \mathrm{~mA}$ 
Figura 62 - Fase da impedância de uma fita GMI de $15 \mathrm{~cm}$ submetida $a \mathrm{i}_{\mathrm{C}}=\left[\mathrm{I}_{\mathrm{CC}}+15 . \operatorname{sen}(2 \pi .10 \mathrm{MHz} . \mathrm{t})\right] \mathrm{mA}$, onde $\mathrm{I}_{\mathrm{CC}}$ assume os valores $0 \mathrm{~mA}, 40 \mathrm{~mA}$ e $80 \mathrm{~mA}$.

Figura 63 - Módulo da impedância de fitas $\mathrm{GMl}$ de $1 \mathrm{~cm}, 3 \mathrm{~cm}$, $5 \mathrm{~cm}$ e $15 \mathrm{~cm}$; submetidas a $\mathrm{i}_{\mathrm{C}}=[0+15 . \operatorname{sen}(2 \pi .10 \mathrm{MHz} . \mathrm{t})] \mathrm{mA} \ldots \ldots .128$

Figura 64 - Módulo da impedância de fitas $\mathrm{GMI}$ de $1 \mathrm{~cm}, 3 \mathrm{~cm}$,

$5 \mathrm{~cm}$ e $15 \mathrm{~cm}$; submetidas a $\mathrm{i}_{\mathrm{C}}=[80+15 . \operatorname{sen}(2 \pi .10 \mathrm{MHz} . \mathrm{t})] \mathrm{mA} \ldots .129$

Figura 65 - Módulo da impedância de fitas $\mathrm{GMI}$ de $1 \mathrm{~cm}, 3 \mathrm{~cm}$,

$5 \mathrm{~cm}$ e $15 \mathrm{~cm}$; submetidas a ic $=[0+15 . \operatorname{sen}(2 \pi .100 \mathrm{kHz} . \mathrm{t})] \mathrm{mA} \ldots . .129$

Figura 66 - Fase da impedância de fitas $\mathrm{GMI}$ de $1 \mathrm{~cm}, 3 \mathrm{~cm}$,

$5 \mathrm{~cm}$ e $15 \mathrm{~cm}$; submetidas a $\mathrm{i}_{\mathrm{C}}=[0+15 . \operatorname{sen}(2 \pi .10 \mathrm{MHz} . \mathrm{t})] \mathrm{mA} \ldots \ldots .130$

Figura 67 - Fase da impedância de fitas $\mathrm{GMI}$ de $1 \mathrm{~cm}, 3 \mathrm{~cm}$,

$5 \mathrm{~cm}$ e $15 \mathrm{~cm}$; submetidas a $\mathrm{i}_{\mathrm{C}}=[80+15 . \operatorname{sen}(2 \pi .10 \mathrm{MHz} . \mathrm{t})] \mathrm{mA} \ldots .130$

Figura 68 - Fase da impedância de fitas $\mathrm{GMI}$ de $1 \mathrm{~cm}, 3 \mathrm{~cm}$,

$5 \mathrm{~cm}$ e $15 \mathrm{~cm}$; submetidas a $\mathrm{i}_{\mathrm{C}}=[0+15 . \operatorname{sen}(2 \pi .100 \mathrm{kHz} . \mathrm{t})] \mathrm{mA} \ldots . .130$

Figura 69 - Valor, ponto-a-ponto, da histerese absoluta, do módulo da impedância, em função do campo magnético, para uma fita GMl de $3 \mathrm{~cm}$ excitada por $\mathrm{i}_{\mathrm{C}}=[80+15 . \operatorname{sen}(2 \pi . f . t)] \mathrm{mA}$, onde f assume os valores $100 \mathrm{kHz}, 750 \mathrm{kHz}$ e $2 \mathrm{MHz}$. 134

Figura 70 - Valor, ponto-a-ponto, da histerese percentual, do módulo da impedância, em função do campo magnético, para uma fita GMI de $3 \mathrm{~cm}$ excitada por $\mathrm{i}_{\mathrm{C}}=[80+15 . \operatorname{sen}(2 \pi$.f.t) $] \mathrm{mA}$, onde f assume os valores $100 \mathrm{kHz}, 750 \mathrm{kHz}$ e $2 \mathrm{MHz}$.

Figura 71 - Valor, ponto-a-ponto, da histerese absoluta, do módulo da impedância, em função do campo magnético, para fitas GMI de $3 \mathrm{~cm}$ e $15 \mathrm{~cm}$ excitadas por $\mathrm{i}_{\mathrm{C}}=\left[\mathrm{I}_{\mathrm{cC}}+15 . \operatorname{sen}(2 \pi .2 \mathrm{MHz} . \mathrm{t})\right] \mathrm{mA}$, onde $I_{\mathrm{CC}}$ assume os valores $0 \mathrm{~mA}$ e $80 \mathrm{~mA}$ 
Figura 72 - Valor, ponto-a-ponto, da histerese percentual, do módulo da impedância, em função do campo magnético, para fitas GMI de $3 \mathrm{~cm}$ e $15 \mathrm{~cm}$ excitadas por $\mathrm{i}_{\mathrm{C}}=\left[\mathrm{I}_{\mathrm{cc}}+15 . \operatorname{sen}(2 \pi .2 \mathrm{MHz} . \mathrm{t})\right] \mathrm{mA}$, onde $\mathrm{I}_{\mathrm{Cc}}$ assume os valores $0 \mathrm{~mA}$ e $80 \mathrm{~mA}$

Figura 73 - Valor, ponto-a-ponto, da histerese absoluta, da fase da impedância, em função do campo magnético, para uma fita GMI de $3 \mathrm{~cm}$ excitada por $i_{\mathrm{C}}=[80+15 . \operatorname{sen}(2 \pi . f . t)] \mathrm{mA}$, onde f assume os valores $100 \mathrm{kHz}, 750 \mathrm{kHz}$ e $2 \mathrm{MHz}$.

Figura 74 - Valor, ponto-a-ponto, da histerese percentual, da fase da impedância, em função do campo magnético, para uma fita GMI de $3 \mathrm{~cm}$ excitada por $i_{\mathrm{C}}=[80+15 . \operatorname{sen}(2 \pi . f . t)] \mathrm{mA}$, onde f assume os valores $100 \mathrm{kHz}, 750 \mathrm{kHz}$ e $2 \mathrm{MHz}$. 136

Figura 75 - Valor, ponto-a-ponto, da histerese absoluta, da fase da impedância, em função do campo magnético, para fitas GMI de $3 \mathrm{~cm}$ e $15 \mathrm{~cm}$ excitadas por $\mathrm{i}_{\mathrm{C}}=\left[\mathrm{I}_{\mathrm{cc}}+15 . \operatorname{sen}(2 \pi .2 \mathrm{MHz} . \mathrm{t})\right] \mathrm{mA}$, onde $\mathrm{I}_{\mathrm{CC}}$ assume os valores $0 \mathrm{~mA}$ e $80 \mathrm{~mA}$

Figura 76 - Valor, ponto-a-ponto, da histerese percentual, da fase da impedância, em função do campo magnético, para fitas GMI de $3 \mathrm{~cm}$ e $15 \mathrm{~cm}$ excitadas por $\mathrm{i}_{\mathrm{C}}=\left[\mathrm{I}_{\mathrm{cc}}+15 \cdot \operatorname{sen}(2 \pi .2 \mathrm{MHz} . \mathrm{t})\right] \mathrm{mA}$, onde $I_{\mathrm{CC}}$ assume os valores $0 \mathrm{~mA}$ e $80 \mathrm{~mA}$

Figura 77 - Características do Módulo de fitas GMI com solda de ponto.

Figura 78 - Características do Módulo de fitas GMI sem solda 140

Figura 79 - Características de Módulo das 5 fitas GMI analisadas, para uma mesma corrente de condicionamento $\mathrm{i}_{\mathrm{C}}=[80+15 \cdot \operatorname{sen}(2 \pi \cdot 100 \mathrm{kHz} \cdot \mathrm{t})] \mathrm{mA}$ 140

Figura 80 - Características da Fase de fitas GMI com solda de ponto.. 141 Figura 81 - Características da Fase de fitas GMI sem solda 
Figura 82 - Características de Fase das 5 fitas GMI analisadas,

para uma mesma corrente de condicionamento

$\mathrm{i}_{\mathrm{C}}=[80+15 \cdot \operatorname{sen}(2 \pi .100 \mathrm{kHz} \cdot \mathrm{t})] \mathrm{mA}$

Figura 83 - Circuito eletrônico de condicionamento e leitura das variações do módulo.

Figura 84 - Circuito eletrônico de condicionamento e leitura das variações de fase

Figura 85 - Fita de $3 \mathrm{~cm}$ submetida a $\mathrm{i}_{\mathrm{C}}=[80+15 . \operatorname{sen}(2 \pi .1 \mathrm{MHz} . \mathrm{t})]$ mA, onde: (a) Módulo da impedância, (b) Fase da impedância, (c) Componente Resistiva e (d) Componente indutiva. 150

Figura 86 - Circuito de transdução de campo magnético em tensão, baseado na variação de fase das fitas GMI, utilizado no transdutor de pressão

Figura 87 - Representação esquemática do transdutor de pressão...... 153

Figura 88 - Variação das componentes resistiva e reativa (indutiva) em função do campo magnético.

Figura 89 - Variação da fase em função da componente resistiva $\left(R_{F}\right)$ e reativa $\left(X_{F}\right)$. (a) Análise tridimensional e (b) Vista superior da análise tridimensional, diretamente associada ao ciclo trigonométrico

Figura 90 - Variação da fase em função da componente resistiva $\left(R_{F}\right)$

e reativa $\left(X_{F}\right)$ por par de quadrantes: (a) $3^{\circ}$ e $4^{\circ}$, (b) $1^{\circ}$ e $2^{\circ}$, (c) $2^{\circ}$ e $3^{\circ}$, e (d) $1^{\circ}$ e $4^{\circ}$ 157

Figura 91 - Generalized Immitance Converter (GIC). 158

Figura 92 - Circuito Frequency-dependent Negative-resistance (FDNR). 159

Figura 93 - Primeira versão da configuração para amplificação da sensibilidade de fase. 
Figura 94 - Características de variação de módulo $(Z)$ e fase $(\theta)$ em função do campo magnético $(H)$, para uma fita $G M I$ de $3 \mathrm{~cm}$ condicionada por uma corrente $\mathrm{i}_{\mathrm{C}}=[80+15 . \operatorname{sen}(2 . \pi . \mathrm{f} . \mathrm{t})] \mathrm{mA}$. Sendo que, em: (a) $f=100 \mathrm{kHz}$, (b) $f=106,3 \mathrm{kHz}$ e (c) $f=107 \mathrm{kHz}$. Ainda, a fita: (a) não está conectada ao circuito de amplificação da sensibilidade de fase e em (b) e (c) está conectada ao circuito de amplificação da sensibilidade de fase. 161

Figura 95 - Segunda versão da configuração para amplificação da sensibilidade de fase.

Figura 96 - Função arco-tangente. 163

Figura 97 - Fita de $3 \mathrm{~cm}\left(\mathrm{Co}_{70,4} \mathrm{Fe}_{4,6} \mathrm{Si}_{15} \mathrm{~B}_{10}\right)$ submetida a $\mathrm{i}_{\mathrm{C}}=[30+15 \cdot \operatorname{sen}(2 \pi .100 \mathrm{kHz} . \mathrm{t})] \mathrm{mA}$, onde: (a) Módulo da impedância, (b) Fase da impedância, (c) Componente Resistiva e (d) Componente indutiva. 167

Figura 98 - Fita de $3 \mathrm{~cm}\left(\mathrm{Co}_{70,4} \mathrm{Fe}_{4,6} \mathrm{Si}_{15} \mathrm{~B}_{10}\right)$ submetida a $\mathrm{i}_{\mathrm{c}}=\left[30+\mathrm{l}_{\mathrm{ac}} \cdot \operatorname{sen}(2 \pi \cdot 100 \mathrm{kHz} \cdot \mathrm{t})\right] \mathrm{mA}$, sendo $\mathrm{l}_{\mathrm{ac}}$ igual a $1 \mathrm{~mA}$ ou 15 mA. Onde: (a) Módulo da impedância, (b) Fase da impedância, (c) Componente Resistiva e (d) Componente indutiva. 168

Figura 99 - Amplificação da sensibilidade de fase: Fase em função do campo magnético. 171

Figura 100 - (a) Componente resistiva (R) de $Z_{\text {sens }}$ em função do campo magnético, e (b) Componente reativa $(X)$ de $Z_{\text {sens }}$ em função do campo magnético. 171

Figura 101 - Fase da impedância de $Z_{\text {sens }}$ em função do campo magnético, para três possibilidades de $\mathrm{R}_{\mathrm{AJ}}: 311,1 \Omega, 312 \Omega$ e $321 \Omega$ 172

Figura 102 - Desenho esquemático do circuito eletrônico do protótipo montado 174

Figura 103 - Detalhes do circuito eletrônico desenvolvido para condicionamento e leitura. 
Figura 104 - Circuito eletrônico simplificado do transdutor magnético GMI desenvolvido, conectado a uma fita GMI (elemento sensor) de $3 \mathrm{~cm}$ disposta no centro de uma Bobina de Helmholtz, utilizada para fins de excitação magnética.

Figura 105 - Saída em tensão do protótipo, com ganho unitário e sem filtragem digital, para um campo magnético senoidal de excitação com amplitude de 0,01 Oe e frequência de $9,8 \mathrm{~Hz}$.

Figura 106 - Saída em tensão do protótipo, com ganho unitário e com filtragem digital, para um campo magnético senoidal de excitação com amplitude de 0,01 Oe e frequência de $9,8 \mathrm{~Hz}$

Figura 107 - Características do ruído 1/f em função da frequência, com zoom na região de: (a) $0,2 \mathrm{~Hz}$ a $150 \mathrm{~Hz}$, (b) $0,2 \mathrm{~Hz}$ a $20 \mathrm{~Hz}$, (c) $4 \mathrm{~Hz}$ a $150 \mathrm{~Hz}$, (d) $75 \mathrm{~Hz}$ a $150 \mathrm{~Hz}$ 177

Figura 108 - Estrutura para leitura gradiômétrica com fitas GMI homogêneas.

Figura 109 - Característica da fase da impedância em função do campo magnético, para as amostras GMI heterogêneas A e B.......185

Figura 110 - Diagrama de blocos do circuito "homogeneizador". 185

Figura 111 - Característica da componente resistiva da impedância em função do campo magnético, para as fitas GMI A e B. 186

Figura 112 - Característica da componente indutiva da impedância em função do campo magnético, para as fitas GMI A e B

Figura 113 - Comparação das características de fase da fita A com as da fita B conectada ao "circuito homogeneizador".

Figura 114 - Erro ponto-a-ponto na região homogeneizada entre as curvas de fase da fita $A$ em relação à fita $B$ conectada ao "circuito homogeneizador". 188

Figura 115 - Comparação das características da componente resistiva em função do campo magnético, entre as fitas: $A, B$ e B conectada ao "circuito homogeneizador" 
Figura 116 - Comparação das características da componente indutiva em função do campo magnético, entre as fitas: $A, B$ e B conectada ao "circuito homogeneizador".

Figura 117 - Representação esquemática da primeira versão da configuração gradiômétrica, com homogeneização e amplificação da sensibilidade de fase.

Figura 118 - Configuração, dos elementos sensores GMI, para leitura triaxial.

Figura 119 - Campo magnético do solenóide $\left(\mathrm{H}_{\text {sol }}\right)$ e suas componentes $\left(\mathrm{H}_{\mathrm{x}}, \mathrm{H}_{\mathrm{y}}\right.$ e $\left.\mathrm{H}_{\mathrm{z}}\right)$, responsáveis pela polarização das fitas $\mathrm{GMI}$. 193

Figura 120 - Esquemático do circuito eletrônico idealizado para o magnetômetro GMI.

Figura 121 - Quantidade anual de patentes (PCT) publicadas sobre magnetoresistência.

Figura 122 - Percentual de patentes (PCT) publicadas, sobre magnetoresistência, por país.

Figura 123 - Quantidade anual de patentes (PCT) publicadas sobre magnetoimpedância 206

Figura 124 - Percentual de patentes (PCT) publicadas, sobre magnetoimpedância, por país. 206

Figura 125 - Representação das Redes Neurais (módulo e fase) utilizadas para estimar as sensibilidades dos elementos sensores GMI 209

Figura 126 - Fluxograma da cadeia de desenvolvimento dos transdutores GMI, com proposição de possíveis trabalhos futuros e aplicações 


\section{Lista de Tabelas}

Tabela 1 - Grandezas magnéticas, suas unidades e suas expressões em termos das unidades de base do SI........................ 46

Tabela 2 - Conversões entre os sistemas de unidades CGS e SI........... 46

Tabela 3 - Comparação do desempenho (fundo de escala e

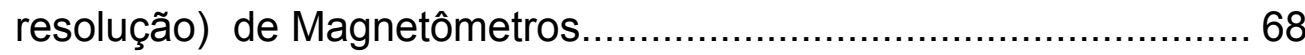

Tabela 4 - Propriedades das Amostras GMI analisadas...................... 98

Tabela 5 - Exemplos de não-correlação entre GMI(\%) e sensibilidade.105

Tabela 6 - Tecnologias de fabricação e suas características..................106

Tabela 7 - Sensibilidades ótimas em função do comprimento das amostras e da corrente de excitação

Tabela 8 - Comparativo das sensibilidades ótimas para fins de homogeneidade.

Tabela 9 - Comparação de desempenho: transdutores baseados na fase versus transdutores baseados no módulo 151

Tabela 10 - Influência de $R_{\text {AJ }}$ na sensibilidade e no fundo de escala.... 173

Tabela 11 - Sensibilidade teórica versus sensibilidade experimental.... 175

Tabela 12 - Características, do circuito eletrônico, ativadas em função dos jumpers.

Tabela 13 - Comparação da sensibilidade dos magnetômetros GMI.... 202 
"If I have seen further it is by standing on the shoulders of giants." "Se pude ver mais longe foi por subir nos ombros de gigantes" Sir Isaac Newton 